\title{
Sick sinus syndrome associated with Erdheim- Chester disease was reversed by interferon-alpha treatment
}

\author{
Xinli Guo ${ }^{*}$, Yuanwei Xu ${ }^{*}$, Ke Wan, and Yucheng Chen
}

Department of Cardiology, West China Hospital, Sichuan University Chengdu, China

Received : February 28, 2021

Revised : March 12, 2021

Accepted: April 5, 2021

\section{Correspondence to} Yucheng Chen, M.D. Tel: +86-18980602149 Fax: +86-28-85422836 E-mail: chenyucheng2003@126.com https://orcid.org/0000-00020601-8039

*These authors contributed equally to this work.
A 64-year-old woman was admitted because of progressive exertional dyspnea for 4 years and recurrent syncope for 2 days. A diagnosis of sinus node syndrome (SSS) was confirmed based on sinus arrest with atrioventricular junctional escape rhythm on electrocardiogram (Fig. 1A). Further cardiac and neurological examinations revealed a mass on right atrium (RA) and pericardial effusion on echocardiography (Fig. $1 \mathrm{~B})$, and a right retro-orbital lesion with normal T1-hypointense enhancement but short T2hypointense enhancement (Fig. 1C) on cerebral magnetic resonance imaging (MRI), indicating Erdheim-Chester disease (ECD). Ancillary imaging examinations further demonstrated typical features of ECD, including the irregular mass on the RA and a right atrio-ventricular groove with relatively shorter $\mathrm{T} 1$ and longer $\mathrm{T} 2$ relaxation times (Fig. 1D) on cardiac MRI, a 'coated aorta' and a 'hairy kidney' (Fig. 1E) on computed tomography, and symmetrical increased uptake of radiotracer in the skull and long bones of the extremities (Fig. 1F) on bone scintigraphy. ECD was finally confirmed by myocardial mass biopsy with typical histological and immunohistochemistry findings of CD68(+), CD1a(-),
S100(-) (Fig. 1G and $1 \mathrm{H}$ ) with negative BRAF ${ }^{\mathrm{V} 600 \mathrm{E}}$ mutation. A single chamber ventricular pacemaker was implanted for SSS treatment, and 3 million units of interferonalpha was administered twice a week for ECD treatment. The patient was pacemaker-dependent at the 2-month follow-up (Fig. 1I), but displayed a restored intrinsic sinus rhythm after 4 months of interferon-alpha treatment (Fig. 1J).

As a non-Langerhans cells histiocytosis with multiorgan involvement, $70 \%$ of patients with ECD have abnormal heart imaging. The most common finding is RA infiltration. In a previous similar case with RA involvement and SSS, an RA lead was implanted in an area with a less thickened wall and no delayed enhancement on cardiac MRI. However, we abandoned the RA approach in consideration of not only the technical difficulties, but also the progression of infiltration that might compromise pacing function. Moreover, the patient experienced SSS remission after interferonalpha treatment, but longer follow-up was needed to observe the long-term effect.

\section{Conflict of interest}

No potential conflict of interest relevant to this article was reported. 


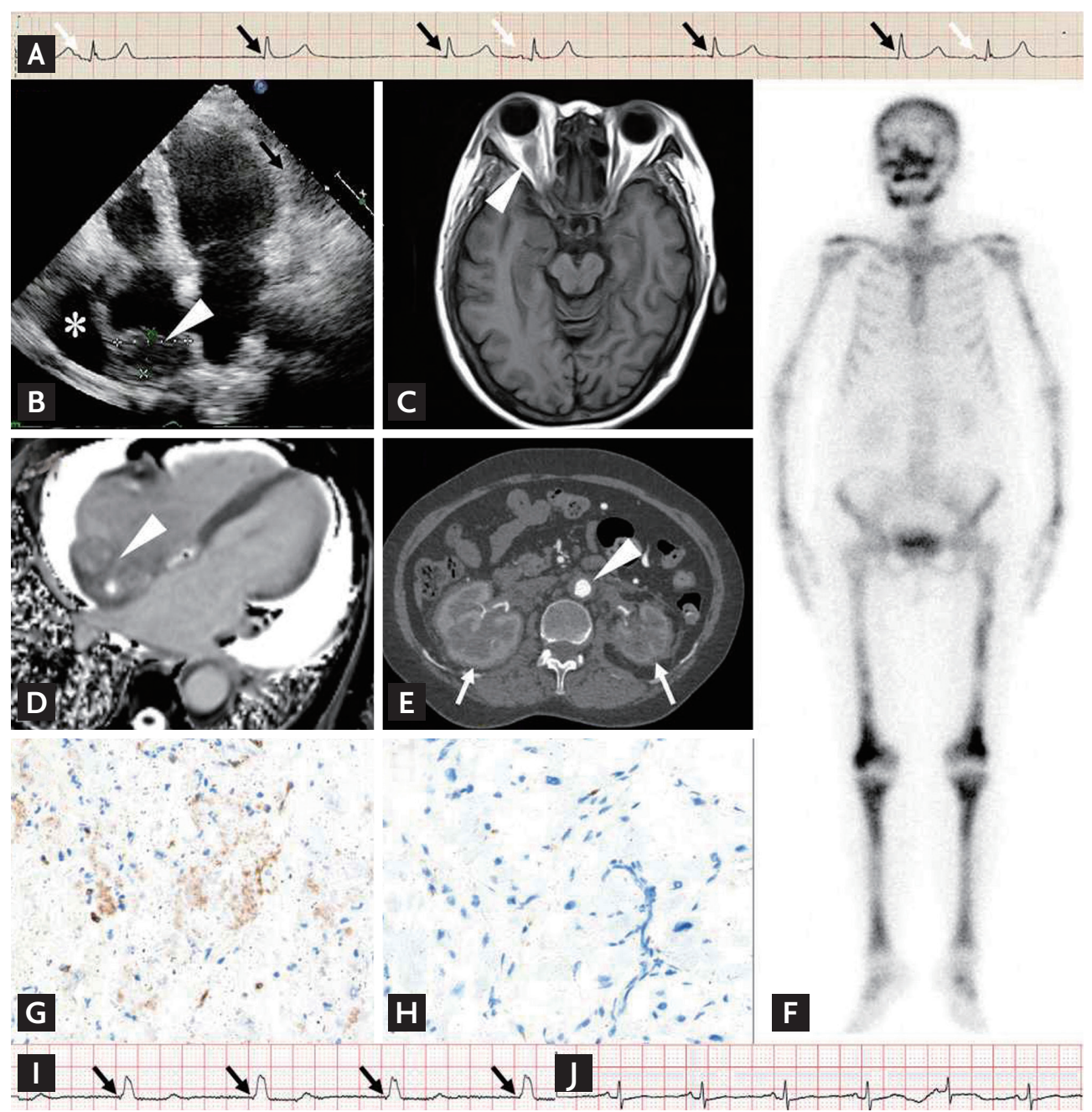

Figure 1. Common features of Erdheim-Chester disease. (A) Sinus arrest with P-P intervals of 4240 ms (white arrows) and atrioventricular junctional escape beat (black arrows) on electrocardiogram. (B) The right atrium (RA) mass (arrowhead) and pericardial effusion (asterisk) on echocardiography. (C) Right retro-orbital abnormal tissue with T2-hypointense enhancement (arrowhead) on cerebral magnetic resonance imaging (MRI). (D) Irregular masses on the RA and right atrio-ventricular groove with T1-hypointense enhancement (arrowhead) on cardiac MRI. (E) 'Hairy kidney' (arrows) and 'coated aorta' (arrowhead) upon enhanced computed tomography. (F) Symmetric longbone hypermetabolism on bone scintigraphy. (G) CD68 (+) and (H) S100 (-) histiocytes on a biopsied specimen of the RA mass. (I) Pacing rhythm with wide and bizarre Q wave, R wave, and S wave (QRS) complex of 50 beats per minutes (pacemaker spikes are marked using black arrows) at 2-month follow-up on electrocardiogram (EKG). (J) Sinus rhythm with 82 beats per minutes on EKG after 4 months of interferon-alpha treatment.

\section{Acknowledgments}

This report was supported by $1 \cdot 3 \cdot 5$ project for disciplines of excellence-Clinical Research Incubation Project, West China Hospital, Sichuan University (ZYJC 18013). 